\title{
2. Integrating knowledge in service delivery-land: a view from The Benevolent Society
}

\begin{abstract}
Annette Michaux
People continually expand their capacity to create the results they truly desire, where new and expansive patterns of thinking are nurtured, where collective aspiration is set free, and where people are continually learning how to learn together. (Senge 1990)
\end{abstract}

\section{Chapter aims}

This chapter offers a practice perspective on three main themes. The first is the importance of learning collaborations and partnerships that move beyond access to knowledge to engagement with and use of learning, and some of the barriers that non-profit practice organisations experience in these interactions. Second, the chapter examines the contribution of non-profit organisations to policy and research due to their connection to communities; and third, the chapter outlines examples of initiatives that illustrate strategies facilitating the mediation of knowledge across sectoral and organisational boundaries.

\section{About The Benevolent Society}

At nearly 200 years old, The Benevolent Society is Australia's oldest non-profit organisation. It is a diverse organisation delivering services to people and communities, with a focus on children and families, older people and women's health. We also run leadership programs bringing people together from business, the community and government for social change. In 2009, services were provided to about 17500 people, and we have approximately 850 staff, 600 volunteers and 350 alumni of our leadership programs. The children's services span out-of-home care, child protection, early intervention, post-adoption resources, long day care and playgroups as well as community development. More information about the organisation can be found at <www.bensoc.org.au $>$ 


\section{Importance of knowledge integration}

The Benevolent Society's purpose is to create caring and inclusive communities and a just society. To do this, we are rethinking how to work and moving the organisation beyond traditional service provision to a stronger focus on linking our work in communities to research and evaluation, as well as policy action (see Figure 2.1).

Figure 2.1 Linking The Benevolent Society's programs and services with research and evaluation as well as policy action

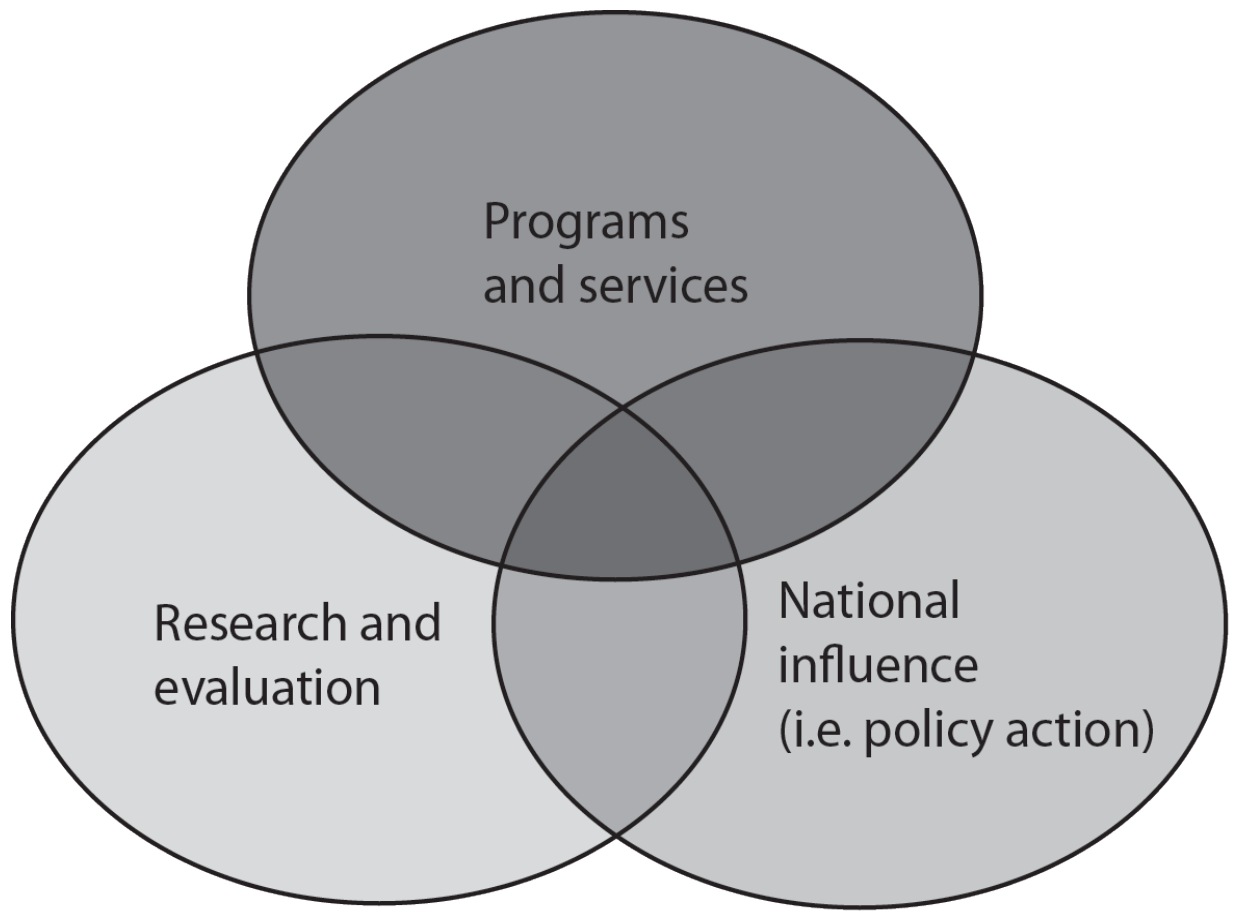

Our purpose calls for us to engage more systematically with researchers and policymakers across all levels of our organisation. Although non-profit organisations are excluded from many policy and research debates, the onus is also on non-profits to make sure we are at the table and that we present wellevaluated and constructive service models and policy ideas for the consideration of policymakers.

Increasingly, non-profit organisations are the recipients of large government contracts for service delivery. This is a positive trend, but programs can encounter difficulties when service providers are excluded from the design of 
programs. Non-profit organisations must be consulted during the design stage of programs, especially when international evidence is being applied to the Australian context.

Community organisations are at the heart of democratic life and civic engagement and are the only institutions with the flexibility and connections to put the policy solutions into practice on the ground.

Attempting to rise to these challenges, non-profit organisations large and small are changing their structures and investing in skills that allow them to play a leadership role at the policy and research table. For example, The Benevolent Society is investing in a comprehensive research and advocacy agenda allowing us to be judicious and strategic in the way we mediate knowledge across internal and external boundaries.

\section{Difficulties of sharing knowledge across sectors}

We find that knowledge is sticky and does not move easily between sectors (Szulanski 1996). In a case study of eight US firms undertaken in the 1990s, it was found that this stickiness was not just about motivation to learn or the greatest barrier to learning across organisations; instead it was found that organisational units did not know how to learn. This important insight forces us to consider learning strategies that will work in the Australian context. We know that the keys to integrating knowledge are people, relationships and interaction combined with strong learning systems, an inter-sectoral culture and good tools to facilitate learning. This requires a shift in our approach to knowledge on a number of levels and is illustrated in Figure 2.2, which I have adapted from Bill Ford's work in Australia on learning organisations.

Ford's work challenges us to look at organisations and how they work both internally and externally. The left side of Figure 2.2 shows how organisations and sectors have traditionally worked. For example, in medicine, a very distinct knowledge hierarchy can exist with medical doctors at the top and patients seen as passive recipients of treatment, rather than partners sharing information about a health problem. The middle of Figure 2.2 forces institutions to radically rethink how they structure their internal operations and relate to external networks if knowledge is to be integrated. The organisational development literature talks of flat structures with fuzzy boundaries where teamwork and collaboration win over traditional hierarchical models of leadership. Crutchfield and McLeod Grant (2008), in a study of successful non-profit organisations in the United States, emphasise that collaborative models are more effective than 
others in forging policy change. The non-profit organisations that have policy impact tend to excel at influencing players outside their boundaries, managing complex networks and relationships through partnerships and coalitions. These organisations tend to have distributed leadership throughout their organisation ensuring that there is high-level engagement with learning how to overcome the social issue at hand.

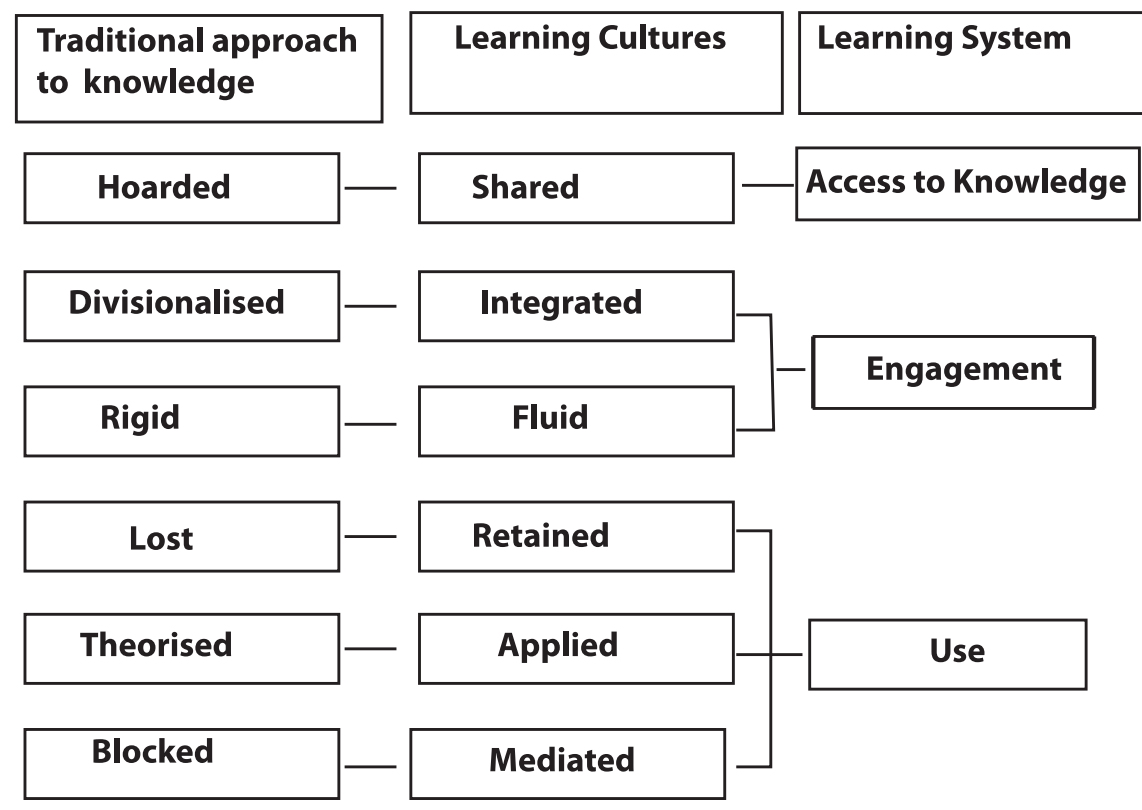

Figure 2.2 Elements involved in moving from traditional approaches to knowledge to learning cultures and learning systems

Adapted from Bill Ford (1999).

The other area of interest to us is the different cultures that arise in different professional groups, as well as the inter-professional hierarchies, with some professions more highly regarded than others. For example, we have observed that early childhood professionals - in particular, those involved in the childcare sector - are often shut out of policy debates about children, reflecting perceptions about their status.

To support knowledge flow across these boundaries, we need to find new, integrated and sustainable systems for bringing people together to learn (the right side of Figure 2.2). The view from service delivery-land is that we have some excellent access to knowledge through a variety of Australian clearing houses and their disseminated publications. We struggle, however, to find ways 
to ensure there is engagement with this knowledge and therefore its translation into use. Later in this chapter, I draw on some examples of how we have moved beyond access at The Benevolent Society.

\section{Why partnerships are important: the contributions of non-profit organisations to policy and research}

Too often those closest to a policy issue or research question are excluded from decision-making processes. Clients and communities on the receiving end of policy as well as the non-profit organisations that are best positioned to respond are not at the table. In Australia, while there is increasing talk of whole-of-government responses, there is no corresponding attention to wholeof-sector responses. Until recently, non-profit organisations were discouraged from advocacy work for social change and sometimes punished and silenced for engaging in policy debates (Hamilton 2007).

The literature on organisational development and partnerships as well as the drive for joint work in the United Kingdom (Ford 1999; Mandell 2006; Anning et al. 2006) show that, to implement what works for children, we have to find new ways of working and learning that are supported by very different institutional arrangements. The community sector could hold the key to some of the more creative and flexible arrangements needed to facilitate this major change and must therefore have a greater voice.

Non-profit organisations play an important role in generating research questions and identifying research gaps. We also have the flexibility to be innovative and responsive and to test new ideas. A growing number of philanthropists who are interested in social change organisations such as The Benevolent Society can organise financial backing for research and learning opportunities that might be considered too risky for government. A recent Australian example of this is the work of the philanthropist Chris Cuffe, who is using innovative approaches to fundraising to generate a steady income stream for the organisation Social Ventures Australia, of which The Benevolent Society and The Smith Family are founding partners (Horin 2008).

Despite the relatively slow start in Australia to engaging philanthropy, it is now poised to play a key role in funding innovation and research and as an additional partner in knowledge integration. Although not the focus of this chapter, this is an exciting development being explored. ${ }^{1}$

1 See, for example, the role of philanthropy in the Pathways to Prevention Project (Homel et al. 2006). 
We are keen to see knowledge flow from practice to research as well as from research to practice. We believe that non-profit organisations can play a significant role as knowledge producers. Framing research questions based on our practice wisdom often generates important insights into the needs of communities and thus informs policy. Our experience in developing a model for working in kinship care has revealed that there is very little Australian research to guide us. We have generated a number of research/policy questions, such as how to measure the outcomes of kinship care work and about good practice models for Indigenous families. We are now working alongside academics and government officials to develop our research agenda together.

Donald Schon's (1983) work is useful as it reveals how professionals know more than they can put into words. His work focuses on how we need to tap into this reflection-in-action and how this vital creativity (or tacit knowledge) might be fostered by organisations.

Schon argues that the best planners, architects, engineers and psychologists cannot always say what they know how to do, but reflective processes are a way of harnessing and sharing this greater problem-solving ability. Good organisations find ways to allow a broader context for reflective inquiry and count it as a legitimate form of professional knowing (Schon 1983).

This process is not easy. The knowledge we hold in community services is often complex, hard to teach and hard to detach from the person who created it. Think of the incredibly complex interactions and processes involved in the way a very good practitioner goes about their work with a family, and in their interactions with that family's community. As Smith (2001) states, this sort of complex know-how cannot just be transmitted; 'it has to be engaged with, talked about and embedded in organisational structures and strategies'. We have found that supervision sessions alongside group learning opportunities such as communities of practice and learning circles open up the creative space for people to engage and share this tacit knowledge. Bringing a wider network to the table during these knowledge-production sessions is an important way for research and policy to connect with communities (see the section on interagency learning forums in this chapter).

Non-profit organisations such as The Benevolent Society have a strong connection to people in their communities and are often in a position to consider community needs as well as strategies for effectively translating research into practice. We are a rich source of case studies and evaluative data about child and family practice. We can also play the role of reminding researchers and policymakers to treat people as participants and partners in processes rather than as objects of concern to 'do things to'. Service delivery organisations such as The Benevolent 
Society are well placed to ensure that research and evaluation in communities can be empowering, enabling processes for participants in which the journey of knowledge generation is as significant as the destination.

\title{
Our limitations in the conversation
}

There is often a gap in the conversation between research, policy and practice. This has been described as the product of their different cultures (Shonkoff 2000; Tsui 2006). Speaking different languages makes communication between sectors difficult. Practitioners can find it hard to articulate research questions and we are sometimes inarticulate about our work and the nature and extent of a problem in a community. We can also lack policy and research literacy and think narrowly about problems, not seeing connections to root causes or larger social issues.

\section{Strategies to facilitate knowledge integration: sector level}

\section{Cross-sectoral project teams, boards and committees}

\begin{abstract}
A well-established mechanism for collaboration includes advisory bodies, inter-sectoral committees and board appointments that ensure cross-sectoral inclusion. The make-up of these bodies should be reflected at different levels of organisations, from the most senior to local implementation levels. The committee layers of the Australian Government's Communities for Children Program are a good example of national to local inter-sectoral attempts at integration. Many project teams and working committees are considering new ways to meet and interact to reflect Australia's need for geographic inclusion including the use of telephone link-ups and moving meeting venues around the country. The Australian Research Alliance for Children and Youth (ARACY) as well as rural health and educational institutions have worked out good systems for such interactions.
\end{abstract}

\section{Secondments and co-locations}

Secondments where academics, policymakers and practitioners are embedded in other organisations are powerful ways to create understanding, integration and more insightful leadership. The Benevolent Society has an arrangement with the NSW Department of Community Services through which staff from each organisation spend a week swapping roles. Feedback tells us that this creates 
understanding and empathy between practitioners. These secondments and direct swaps have been known to take place between senior bureaucrats and academics. The Brotherhood of St Laurence, a service provider in Victoria, has several academics working between the university and the agency over the long term.

Co-locating staff from different agencies can also bring benefits (Department for Education and Skills 2003); however, our experience is that this needs to be carefully designed and that co-location alone does not produce integration, as communication problems can persist (Anning et al. 2006). Teams must be actively encouraged to work more closely. We have found that project-based work can facilitate this. Sometimes community members find that co-location does not work for them. For example, locating child protection services in schools can be highly stigmatising for parents required to visit the onsite 'welfare'.

\section{Cross-sectoral forums}

Workshops and symposia that bring policymakers and researchers together would be richer if they more regularly included and facilitated insights from skilled practitioners and community members. While this requires some planning and additional support, our experience is that the views of practitioners and community members sometimes need to be sought in more creative ways than happens in traditional modes of inquiry. We have used interview techniques to gain practitioner insights and creative tools such as artworks to understand children's reflections on a particular topic or social issue. Less formal opportunities to meet and talk also include using community festivals and exhibition-style booths where people can approach one another without feeling they are having public conversations.

\section{Knowledge-brokering organisations}

The ARACY and the Sax Institute (a NSW-based organisation that develops research products and builds partnerships between researchers and health policy and service delivery agencies for better health) are dedicated to bringing together practice, policy and research for better outcomes. Our experience of both entities is that they are facilitating new opportunities for non-profit organisations such as The Benevolent Society to be more closely involved in policy debates. This has taken the form of being invited to speak at various forums, as well as introductions to new networks and individuals, especially in the policy domain, which can be difficult for outsiders to access. 


\section{Boundary-breaking activities}

Service delivery organisations that play a role in undertaking research or in policy development help break down barriers and ensure that new and more grounded perspectives are included in decision-making processes. Increasingly, the large non-profit organisations have their own research and policy analysis capacity. In New South Wales - following the lead of Victorian community organisations - we are regularly meeting to develop research themes and ideas across community agencies. For example, The Benevolent Society initiated a non-government research forum in New South Wales. In addition, the University of Sydney's Faculty of Education and Social Work has played an important role in exchanging knowledge with community services.

\section{Knowledge-integration techniques: practice perspectives}

Research shows that face-to-face contact is the best way to get evidence into the hands of those who need it (Reardon et al. 2007). We have certainly found this to be the case. Some research centres offer great promise in their responsiveness to our questions about messages from research. The following examples show some useful strategies for linking practice to research and policy.

\section{The Benevolent Society's evidence-based parenting programs}

This project aims to implement evidence-based parenting programs across our children's services. It has involved a number of challenging steps and forced us to engage with, and make decisions about, evidence and then implement a new way of working across a large and geographically diverse organisation. We have learnt that a staged approach to practice change is required - that is, one that takes account of access, engagement and use of knowledge. This example highlights the interaction of good people and good processes, as well as systems to implement evidence in the world of practice.

Our first and most important step in 2003 was for the management team to commit to implementing evidence-informed practice across our child and family services. This has involved some major change-management challenges. The next step was accessing the research, in order to understand and engage with it. We approached the Parenting Research Centre from Victoria to run workshops for our services on evidence-based parenting skills programs. This centre's links 
to practice enabled them to successfully bridge the research-to-practice divide as they spoke the same clinical language as our practitioners and helped us develop our practice in a way that made sense.

Another key ingredient was the knowledge-brokering role of one senior manager, who used a Churchill Fellowship to study parenting programs overseas. His knowledge and commitment meant we had a strong internal champion for the implementation process. This manager has been in a position to seek advice from international experts on the program and consider its application to rural and remote Australia including Indigenous families.

The next step was systematic implementation of the Incredible Years parenting program. Organisational processes needed to be shifted to foster a culture of performance around evidence-informed practice (Letts et al. 1999). This included building outcomes around the implementation into business plans, recruitment of managers with knowledge-brokering skills and improving performanceappraisal systems. We are also developing practice guidelines and making explicit the roles of managers in implementing evidence-informed practice.

This experience has helped us better understand our role in knowledge brokering. It confirms the need for designated internal staff to carry a project forward as well as strong links with the external research and practice community internationally. We have found that in practice-land we need to engage tenaciously with new knowledge if it is to get a foothold in use and practice change.

\section{Using seminars as a springboard to practice change}

Another example is a seminar on best practice in child and family work presented by the Centre for Community Child Health. The seminar was held at The Benevolent Society and we found that when we had a critical mass of managers and practitioners in the room to engage in a dialogue, we started to see practice shifts taking place as a result. This could be for several reasons, including enough people hearing the same, very well-presented message, which means they can follow it through more easily as enough people get it, talk about it and use it.

\section{Inter-agency learning forums}

Sometimes face-to-face contact is not possible. The Central Coast Networks of Practice (funded by Families New South Wales) offers a research-to-practice clearing house and themed practitioner newsletters. These are important regional avenues for translating research into practice, as well as for the sharing 
of practice wisdom. These practice networks also enable conversations between practitioners from different disciplines. The network takes the work of a number of Australian clearing houses and tailors it to the Central Coast audience. This has been a very effective way of increasing access to research.

Linked to Networks of Practice, but more localised, is the 2261 Services Networking Project (funded through the Commonwealth Government's Communities for Children initiative). The larger Communities for Children advisory committee was devoting little time in meetings to focusing on knowledge sharing. Although managers were able to attend some of the 'webinars' and read topical papers (knowledge-sharing tools offered through ARACY), local practitioners were not accessing these resources. A more responsive local effort was needed. The 2261 Project meets and discusses topics such as behaviour management and transition to school when there are specific practice questions. The group then discusses the issues, shares ideas and brings in speakers. The emphasis is on working through issues in a timely fashion and through informal sharing.

We have been running a strengths-based practice project (in partnership with Gowrie, Sydney, another children's service provider, funded by Families New South Wales) with a reach of 450 early childhood services. Designed by staff with excellent knowledge-brokering skills and strong links to research, the project includes broad-based dissemination as well as an intensive component working with up to 45 practitioners. Evidence on early childhood development is translated into formats and tools that early childhood workers can understand, which allows the group of 45 to engage in learning circles and other reflective learning spaces to discuss implementing new practices.

The evaluation findings show that the combination of creating accessible material and learning spaces is a powerful way of integrating knowledge. Two focus group participants reflected on their experiences as follows:

It was the most beneficial training for Children's Service managers which I have done in 25 years as a director of a service. I wish that all new directors could be offered up to 12 months of facilitated SBP learning circles. It would save them many years of learning through trial and error.

I was rather sceptical in the beginning and not sure that I would have the time to commit to the project but I am so glad I did as it has given me the tools and skills to approach work with staff, families and children with some validation. 


\section{Partnerships in community projects}

Initiatives such as the Australian Government's Communities for Children initiative provide excellent opportunities for cross-sectoral and crossdiscipline engagement through a range of local projects. They bring to life the complexities and rewards of deep engagement in community settings. They involve partnerships between community organisations, community members, social policy researchers (in this case, the University of NSW Social Policy Research Centre), local evaluators (on-the-ground researchers) and state and Commonwealth officers, with connections to organisations such as ARACY and the Australian Institute of Family Studies. Our particular Communities for Children projects (located in south-western Sydney and the NSW Central Coast) have used a local collaborative structure to take up the challenge of designing community initiatives that are evidence based and responsive to local need.

Attempts at true collaborations - that is, those efforts in which participants come together to solve a complex issue and are interdependent - are resource intensive and require strong leadership. This involves people entering into new ways of thinking, working and making changes to existing systems and structures (Mandell 2006). For example, the engagement process for the Communities for Children projects was time consuming (about 18 months of planning when the original time line was six months) and required highly skilled managers. This highlights that bringing a Commonwealth strategy to life on the ground with the added central ingredient of community participation often involves hidden and unacknowledged street-level knowledge brokering. Policy initiatives that require this level of cross-sectoral and community involvement can have unrealistic expectations and be conceptually undercooked. The evaluative work on these programs will help us better understand how to successfully negotiate such partnerships.

\section{Partnerships in research}

We have had several experiences partnering across policy, practice and research through Australian Research Council (ARC) Linkage grants. These grants can facilitate knowledge integration in the following ways

- Increased respect and the breaking down of stereotypes, as the process of deep involvement in a research project allows collaborators to understand the strengths that they and others bring, as well as appreciating the constraints that others are working under.

- Promoting understanding of organisational context. For example, in our experience, bureaucracies change over the course of the project in a way that other partners do not. Staff turnover is high and decisions must go through 
hierarchical processes. We have learnt to expect high turnover of personnel and the need for excellent documentation around roles, responsibilities and sign-off for the project, so that it does not drift from the original aims.

- Gaining buy-in for dissemination and implementation of research findings from partners (this is why it is important to have policy officials as partners in the projects). A project being undertaken with Queensland and Sydney universities, the Queensland Government Treasury and Mission Australia ensured that senior bureaucrats were involved in initially framing the research questions to try to ensure relevance to policy.

- Allowing 'younger' and 'older' generations to work together. Older members of the team bring wisdom and experience and younger members bring fresh ideas. Connecting young researchers and policy officers is also paving the way for long-term inter-sectoral and cross-disciplinary work in the next generation.

- Involving practitioners from the partner organisation in the research, enabling them to learn valuable skills and competencies. This involvement has led to the partner organisations having greater ownership of the results.

- Having the right people at the table to comment and correct, so that documents about the research results can be designed more effectively and timed to meet the needs of the different sectors.

- Seeking research-practice linkage projects, such as those funded by the ARC, to offer opportunities for boundary-breaking roles in non-research organisations. As a practice organisation, we have been able to employ early career researchers, as linkage projects allow for publication, presentation and student supervision opportunities alongside a deeper connection to research application. Collaborations tend to seep to other sections of organisations keen to communicate research findings. Staff members in other parts of partner organisations, such as media and public relations officers, have also enjoyed the benefits of working jointly (with researchers) on media releases and other communication strategies.

\section{Summary of challenges and concluding thoughts}

The examples in this chapter show a number of promising initiatives for better integrating knowledge. They also point to some of the many challenges.

Australia has developed some excellent knowledge-dissemination mechanisms, especially our clearing houses (for example, the four Australian Institute of Family Studies clearing houses - on different topics - and the Domestic and Family Violence Clearing House). As trusted sources of evidence, clearing 
houses create a firm base to build from. Australia is, however, behind many other Western nations in taking the next step to integrated cross-sectoral processes and structures to allow people to engage with and therefore use this knowledge. This is particularly challenging in a country of this size. We estimate that Benevolent Society practitioners have about five minutes a day of reading time. It is therefore unlikely that they will be able to engage with the written evidence unless we find the right systems and new ways to resource the mediation of it.

The differing time scales needed for research continue to hamper evidenceinformed policy and practice. The need for rapid answers to practice and policy questions is often out of step with the nature of research grant funding and the operation of academic institutions, meaning that it is often hard to be responsive in this environment (for example, ARC Linkage grant funding processes are slow). Research and evaluation consultants can be brought in to fill this gap and we need to find ways to ensure these private providers contribute to knowledge integration.

Another constraint is the lack of recognition that academic institutions give for the kind of policy and practice research publications and activities (plain English and brief) that are useful to the field. We understand that academics face significant constraints to being involved in many of the sorts of projects that we would find useful. Academics find their important work with practitioners goes unrecognised unless it can be made into a journal article.

Collaboration in developing and undertaking the research is the first step in a process that needs to extend to implementing the findings through the policy process. There is often pressure for academics to cease their involvement when the research is complete. We need to find ways to fund follow-up activity to look at how to get research knowledge into the policy process.

Unlike researchers based in universities, the policy and practice workforce tends to be more transient, moving across agencies, departments and roles. This mobility presents constant challenges to collaborative efforts and can seriously undermine longer-term projects when a project champion moves on.

Perhaps the biggest hurdle remains the policy community's struggle to find effective ways to engage community members and community organisations. Until we engage with those at the receiving end of policy implementation, we will miss vital information about what works in Australia.

Despite the challenges outlined, I have illustrated a number of promising examples of knowledge integration. At the policy level, the new focus on social inclusion in Australia is a welcome sign that cross-sectoral efforts will guide our approach to complex social and economic issues. 
We are keen to know if collaborative efforts and initiatives are making a tangible difference to children and their families. Mapping a path between knowledge brokering and better policies and practices is not easy, but it is very important that we evaluate the extent to which these efforts are achieving results.

The organisational development and knowledge integration literature (Bammer et al., this volume) points to the need for new institutional arrangements that allow for integrated learning between research, policy and practice, as well as real changes to how we roll out policies and deliver services to children and their communities. If knowledge is sticky, hoarded and theorised, it is our job as a large service provider to play a leadership role in making it runny, accessible and useable. The examples used in this chapter show it can be done. Now is the time to take our efforts to scale!

\section{References}

Anning, A., Cottrell, D., Frost, N., Green, J. and Robinson, M. 2006, Developing Multiprofessional Teamwork for Integrated Children's Services, Open University Press, Australia.

Crutchfield, L. and McLeod Grant, H. 2008, Forces for Good. The six practices of high-impact nonprofits, Jossey-Bass, San Francisco.

Department for Education and Skills 2003, Every Child Matters, Government of the United Kingdom, London.

Ford, B. 1998, 'Mediating knowledge', HR Monthly, September.

Ford, B. 1999, Diagrams on learning organisations, Unpublished ms.

Hamilton, C. 2007, Silencing Dissent, Allen \& Unwin, Sydney.

Homel, R., Freiberg, K., Lamb, C., Leech, M., Carr, A., Hampshire, A., Hay, I., Elias, G., Manning, M., Teague, R. and Batchelor, S. 2006, The Pathways to Prevention Project: The first five years, 1999-2004, Mission Australia and Key Centre for Ethics, Law, Justice and Governance, Griffith University, Brisbane.

Horin, A. 2008, 'The \$33m man is back-but now he's working for free', Sydney Morning Herald, 21 July 2008.

Letts, C. W., Ryan, W. P. and Grossman, A. 1999, High Performance Nonprofit Organisations. Managing upstream for greater impact, John Wiley \& Sons, USA. 
Bridging the 'Know-Do' Gap

Mandell, M. 2006, Do networks matter: the ideals and realities, Keynote address to Government and Communities in Partnership Conference, Centre for Public Policy, University of Melbourne, 25-27 September 2006, viewed 5 May 2010, <http://www.public-policy.unimelb.edu.au/conference06/>

Michaux, A. 2006, Learning Organisations and Why They Are Important in Community Services, The Benevolent Society, Paddington, NSW, viewed 5 May 2010, <www.bensoc.org.au>

Reardon, R., Lavis, J. and Gibson, J. 2007, 'From research to practice: a knowledge transfer planning guide', Insight and Action, issue 1 (March), Canadian Health Services Research Foundation, Ottawa.

Saunders, P. and Walter, J. 2005, Ideas and Influence. Social science and public policy in Australia, UNSW Press, Sydney.

Schon, D. 1983, The Reflective Practitioner: How professionals think in action, Basic Books, New York.

Senge, P. 1990, The Fifth Discipline. The art and practice of the learning organization, Random House Australia, Sydney.

Shonkoff, J. P. 2000, 'Science, policy and practice: three cultures in search of a shared mission', Child Development, vol. 71, no. 1, pp. 181-7.

Smith, M. 2001, 'Chris Argyris: theories in action, double loop learning and organisational learning', The Encyclopedia of Informal Education, viewed 5 May 2010, <www.infed.org/thinkers/argyris.htm>

Szulanski, G. 1996, ‘Exploring internal stickiness: impediments to the transfer of best practice within the firm', Strategic Management Journal, vol. 17.

Tsui, L. 2006, A Handbook on Knowledge Sharing: Strategies and recommendations for researchers, policymakers, and service providers, Community University Partnership for the Study of Children, Youth and Families, Alberta. 